\title{
Crescimento da ostra Crassostrea rhizophorae cultivada em diferentes densidades de estocagem no Litoral Norte de Pernambuco
}

\author{
Oyster Crassostrea rhizophorae Growth farmed at \\ different stocking densities in north coast of Pernambuco
}

'Laboratório de Maricultura Sustentāvel (LAMARSU)

Departamento de Pesca e Aquicultura Universidade Federal Rural de Pernambuco (UFRPE), Av. Dom Manoel de Medeiros, s/n, Dois Irmãos, CEP 52171-900 Recife, PE, Brasil ${ }^{2}$ Escritôrio Regional da llha de Itamaracá, Instituto Agronômico de Pernambuco (IPA)

Ilha de Itamaracă, PE, Brasil

* autor correspondente 凶leonidasocjr@hotmail.com
Leônidas de Oliveira Cardoso Junior ${ }^{*}$, Henrique David Lavander ${ }^{1}$, Sergio Rodrigues da Silva Neto ${ }^{\text {, }}$ Andrē Batista de Souza', Luis Otavio Brito da Silva², Alfredo Olivera Gálvez
RESUMO: O presente trabalho avaliou o crescimento da ostra do mangue Crassostrea rhizophorae em diferentes densidades de estocagem. A espécie foi cultivada utilizando-se o método de balsa por 105 dias, no estuário do rio Itapessoca, litoral norte de Pernambuco. O desenho experimental considerou três tratamentos com cinco repetições cada, sendo os tratamentos representados pelas densidades de 400 ostras $/ \mathrm{m}^{2}$ (Tratamento A), 800 ostras $/ \mathrm{m}^{2}$ (Tratamento B) e 1200 ostras $/ \mathrm{m}^{2}$ (Tratamento C). Foram utilizados ANOVA e o teste de Tukey $(\mathrm{P}<0,05)$ para análise estatística. $\mathrm{O}$ comprimento médio inicial das sementes das ostras foi de $24,8 \mathrm{~mm}$ e final de $41,3 \pm 4,8 \mathrm{~mm}, 39 \pm 5 \mathrm{~mm}$ e 38,4 $\pm 4,2 \mathrm{~mm}$ para os tratamentos A, B e C, respectivamente, havendo diferença significativa para o crescimento final em comprimento para as diferentes densidades.

PALAVRAS-CHAVE: Ostreicultura, maricultura, bivalve.
ABSTRACT: This study evaluated the growth of mangrove oyster Crassostrea rhizophorae at different stocking densities. The species was cultivated through the raft method for 105 days in the estuary of the 'Itapessoca' River, in the northern coast of the State of Pernambuco. The research design comprised three treatments with five replicates each, represented by the following densities: 400 oysters $/ \mathrm{m}^{2}$ (Treatment $A$ ), 800 oysters $/ \mathrm{m}^{2}$ (Treatment $B$ ) and 1,200 oysters $/ \mathrm{m}^{2}$ (Treatment $C)$. ANOVA and Tukey test $(P<0.05)$ were used for statistical analysis. The average length of the initial oyster seed was $24.8 \mathrm{~mm}$; the final lengths were $41.3 \pm 4.8 \mathrm{~mm}, 39 \pm 5 \mathrm{~mm}$, and $38.4 \pm 4.2 \mathrm{~mm}$ for treatments $A, B$ and $C$, respectively, showing significant difference in KEYWORDS: Oyster culture, mariculture, bivalve. the final length growth for the different densities.

\section{Introdução}

Um dos fatores limitantes para o crescimento e desenvolvimento da população mundial é a disponibilidade de alimento (FOOD..., 2004). Alimentos com alto valor nutritivo como os de origem aquática, advindos da pesca e aquicultura, são importantes para a segurança alimentar mundial, pois representam $15,3 \%$ do total de proteína animal consumida no mundo (FOOD..., 2009). O fornecimento aparente de pescados per capita para consumo humano em 2009 foi de 17,1 kg e seu crescimento é atribuído à aquicultura, uma vez que a produção mundial por captura vem se mantendo estável nos últimos anos (FOOD..., 2010). 
Segundo dados da FAO (FOOD..., 2010), os principais ramos da aquicultura mundial são: o cultivo de peixes de água doce, macroalgas e moluscos. Os moluscos representam o terceiro maior grupo de animais cultivados mundialmente, com 13,1 milhões de toneladas produzidas em 2008. Neste grupo, as ostras representam $31,8 \%$, bivalves de sedimento $24,6 \%$, mexilhões $12,4 \%$ e vieiras $10,7 \%$.

Segundo dados do Cepene (CENTRO..., 2008), as capturas de moluscos durante o ano de 2006, em Pernambuco, representaram $30,2 \%$ do total de pescado capturado pela pesca artesanal, equivalente a 4.229,5 toneladas, tendo as ostras representado 6,6\%. Devido à importância deste molusco para os pescadores artesanais, o cultivo pode ser uma importante ferramenta na geração de renda e redução na exploração desta espécie em áreas estuarinas.

As variáveis que afetam os sistemas de cultivo de moluscos têm sido estudadas por vários pesquisadores, como densidade de estocagem, estrutura de cultivo, frequência de manejo entre outras (PEREIRA et al., 2001; ALBUQUERQUE; FERREIRA, 2006).

O presente trabalho teve como objetivo avaliar o crescimento da ostra do mangue cultivada em diferentes densidades de estocagem.

\section{Material e Métodos}

\section{Localização da área de estudo}

O estudo foi realizado no estuário do rio Itapessoca $\left(7^{\circ} 40^{\prime}\right.$ 31.09" S e $034^{\circ}$ 50' 41.78” W), no município de Goiana, Litoral Norte do Estado de Pernambuco, distante cerca de $80 \mathrm{~km}$ de Recife (Figura 1). O estuário faz parte do Sistema Estuarino de Itamaracá (SEI), que abrange mais outros seis pequenos rios e possui uma bacia hidrográfica que abrange cerca de $730 \mathrm{~km}^{2}$ (MEDEIROS; KJERFVE, 1993).

\section{Desenho experimental}

$\mathrm{O}$ experimento foi inteiramente casualizado com três tratamentos: 400 ostras $/ \mathrm{m}^{2}$ (tratamento A), 800 ostras $/ \mathrm{m}^{2}$ (tratamento B) e 1200 ostras $/ \mathrm{m}^{2}$ (tratamento C) e cinco

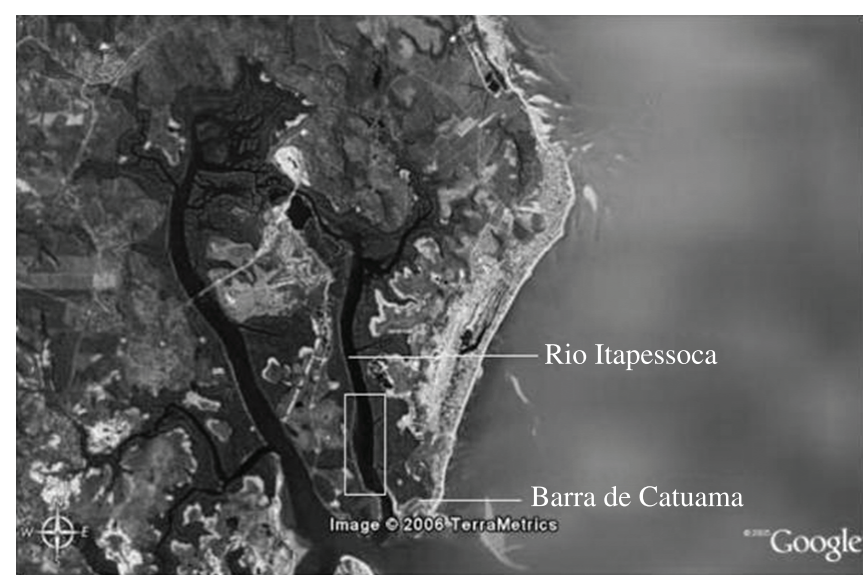

Figura 1. Localização das unidades produtivas de ostra no estuário do Rio Itapessoca (7०40’31.09”' S e 034 50'41.78” W). repetições, perfazendo quinze unidades experimentais (Tabela 1), sendo cada unidade experimental constituída por uma lanterna com cinco andares.

\section{Transporte e aclimatação das sementes de ostra}

As sementes foram adquiridas na Associação de Aquicultores de São Cristóvão, localizada em Sergipe, onde foram coletadas em ambiente natural, utilizando-se, para isso garrafas plásticas (pets) recortadas e sobrepostas, que serviram de substrato para a fixação das larvas, que foram selecionadas por tamanho e transportadas em caixas de isopor, sem água, apenas envolvidas por esponjas umedecidas com o propósito de evitar o ressecamento.

Para a aclimatação das sementes, foram utilizados aquários com capacidade de $160 \mathrm{~L}$, nos quais foram acondicionadas em estruturas de lanternas. Durante todo o período de aclimatação a água recebia aeração por meio de um soprador. O tempo de aclimatação foi de 2 horas, levando-se em conta as diferenças de salinidade e temperatura, considerando-se uma parte de salinidade e $2{ }^{\circ} \mathrm{C}$ de temperatura por hora.

Depois da aclimatação, foi realizado o povoamento nas estruturas de cultivo. O comprimento $(\mathrm{mm})$ e a largura $(\mathrm{mm})$ inicial das ostras nos tratamento foram, respectivamente, $24,8 \pm 0,7$ e $14,7 \pm 4$ (tratamento A), $24,8 \pm 0.55$ e $14,7 \pm 4,3$ (tratamento B) e $24,8 \pm 0.45$ e $14,7 \pm 3,9$ (tratamento C). O tempo de cultivo foi de 105 dias.

\section{Instalações e manejo de cultivo}

As ostras foram cultivadas em sistema de balsa, em parceria com a comunidade de pescadores de Barra de Catuama, município de Goiana, PE. As balsas são estruturas retangulares com área útil de $16 \mathrm{~m}^{2}$, compostas de madeira, cabos, flutuadores e âncora, ligados entre si, de forma a manter as ostras submersas no volume de água. Abaixo das balsas são colocadas as lanternas, que são estruturas cilíndricas e teladas com uma abertura de malhas de $1 \mathrm{~mm}$, com cinco andares de $20 \mathrm{~cm}$ de altura e $40 \mathrm{~cm}$ de diâmetro (Figura 2).

As lanternas, juntamente com as ostras, foram limpas a cada 15 dias e delas eram retirados o sedimento e os organismos incrustantes, com auxílio de escovas, espátulas e também exposição ao sol, no intuito de reduzir possíveis competidores, parasitas e algas periféricas.

\section{Variáveis de produção}

Quinzenalmente, foram realizadas as medidas biométricas, através do uso de um paquímetro com precisão de $0,05 \mathrm{~mm}$, em uma amostra de $20 \%$ de ostras de cada unidade experimental.

Tabela 1. Densidades de estocagem das sementes Crassostrea rhizophorae para os tratamentos A. B e C.

\begin{tabular}{ccc}
\hline \multirow{2}{*}{ Tratamentos } & \multicolumn{2}{c}{ Densidade de estocagem } \\
\cline { 2 - 3 } & Ostras/andar & Ostras $/ \mathbf{m}^{\mathbf{2}}$ \\
\hline A & 50 & 400 \\
B & 100 & 800 \\
C & 150 & 1200 \\
\hline
\end{tabular}




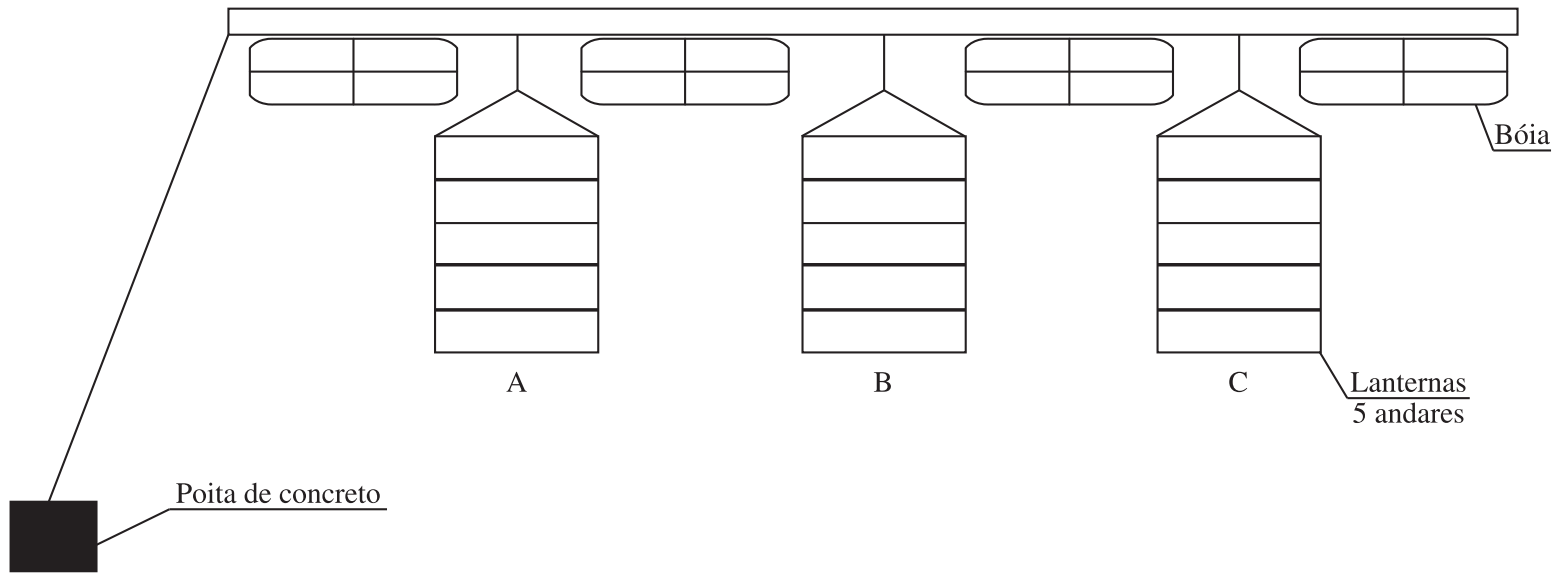

Figura 2. Estrutura de balsa e lanternas utilizadas no cultivo de ostras.

As medidas utilizadas foram comprimento e largura, sendo o comprimento correspondente à máxima dimensão entre a região dorsoventral, e a largura corresponde à distância máxima entre o eixo anteroposterior (GALTSOFF, 1964).

A taxa de sobrevivência das ostras ( $\mathrm{S}$ ) também foi verificada quinzenalmente, sendo calculada pela seguinte fórmula:

$\mathrm{S}=\mathrm{NT} /(\mathrm{No} \times 100)$

Onde: $\mathrm{S}=$ sobrevivência $(\%) ; \mathrm{NT}=$ Número de indivíduos sobreviventes ao final do período avaliado; No = Número inicial de indivíduos colocados nas lanternas.

\section{Análises estatísticas}

Os resultados foram submetidos ao teste de Shapiro-Wilk para verificar a normalidade dos dados e, posteriormente analisados, através de ANOVA e teste de Tukey $(\mathrm{P}<0,05)$. Para análise, foi utilizado o programa Statistica, versão 8.

\section{Resultados e Discussão}

Durante 105 dias de cultivo no tratamento A ( $\left.400 \mathrm{ostras} / \mathrm{m}^{2}\right)$, as ostras obtiveram uma taxa de crescimento mensal médio em comprimento de $5,53 \mathrm{~mm}$ e em largura de $5,36 \mathrm{~mm}$, enquanto os tratamentos B ( 800 ostras $\left./ \mathrm{m}^{2}\right)$ e C $\left(1200\right.$ ostras $\left./ \mathrm{m}^{2}\right)$ apresentaram um crescimento médio de comprimento de $4,73 \mathrm{~mm}$ e $4,53 \mathrm{~mm}$ e de largura de $5,23 \mathrm{~mm}$ e 4,73 $\mathrm{mm}$, respectivamente.

O comprimento $(\mathrm{mm})$ e largura $(\mathrm{mm})$, média final, no tratamento A (400 ostras $/ \mathrm{m}^{2}$ ) foram $41,3 \pm 4,8$ e $30,8 \pm 4,5 ;$ no B $\left(800\right.$ ostras $\left./ \mathrm{m}^{2}\right)$, foram $39 \pm 5$ e $30,4 \pm 4,7$; e no C $\left(1.200\right.$ ostras $\left./ \mathrm{m}^{2}\right)$, foram $38,4 \pm 4,2$ e $28,9 \pm 4,4$, respectivamente, havendo diferença significativa entre os tratamentos (Figuras 3 e 4).

Os estudos de crescimento em ostras apresentam diferenças nos resultados. Santos (1978), em experimentos realizados na Bahia, encontrou, para o crescimento da C. rhizophorae, valor médio de 0,64 mm/mês, e (AKABOSHI; CHAGAS SOARES, 1988), cultivando no Canal de Bertioga, Crassostrea brasiliana com tamanho médio inicial de 32,25 mm, obtiveram, após 12 meses, um comprimento médio final de $60,5 \mathrm{~mm}$, com um crescimento mensal de 5,04 mm. Já Littlewood (1991) registrou a ocorrência de crescimento em $C$. rhizophorae de comprimento e largura que variou entre $12,6 \mathrm{~mm} / \mathrm{mês}$ e $15 \mathrm{~mm} / \mathrm{mês}$ e $3 \mathrm{~mm} / \mathrm{mês}$ e $6 \mathrm{~mm} / \mathrm{mês}$, em Cuba e Porto Rico, respectivamente.

Pereira et al. (2001), avaliando o crescimento da ostra Crassostrea brasiliana, obteve crescimento mensal de 2,64 mm, 2,16 mm e 2,18 mm, respectivamente, nos locais Porto, Retiro e Mandira, na região estuarina de Cananeia, São Paulo. MacCacchero, Ferreira e Guzenski (2007), cultivando a ostra Crassostrea sp. na praia de Sambaqui em Santa Catarina, obtiveram taxas de crescimento de $9,9 \mathrm{~mm} / \mathrm{mês}$, durante 5 meses de cultivo.

Modesto et al. (2010), avaliando o crescimento de C. rhizophorae em lagoa de tratamentos de efluentes de viveiros de fazenda de camarão, obtiveram crescimento mensal de $4,3 \mathrm{~mm}, 4,4 \mathrm{~mm}$ e $4,5 \mathrm{~mm}$, nas densidades de 250 ostras $/ \mathrm{m}^{2}$, 500 ostras $/ \mathrm{m}^{2}$ e 750 ostras $/ \mathrm{m}^{2}$, respectivamente.

Os dados de crescimento obtidos nesse experimento estão semelhantes aos encontrados por Modesto et al. (2010) e Akaboshi, Chagas Soares (1988), porém diferem dos encontrados por Santos (1978), Littlewood (1991), Pereira et al. (2001) e MacCacchero, Ferreira e Guzenski (2007).

No último mês de cultivo, obteve-se um crescimento das ostras de forma mais acentuada que nos meses anteriores, marcados por intensa precipitação pluviométrica, acarretando uma acentuada variação da salinidade e gerando grande quantidade de sedimento em suspensão. Segundo Imai (1977) a quantidade de sedimento em suspensão é um fator limitante para o cultivo de ostras, pois afeta sua respiração e alimentação, causando a redução ou total paralisação no ritmo de crescimento.

De acordo com Johnscher-Fornasaro (1981), a baixa salinidade é outro fator prejudicial ao crescimento da Crassostrea rhizophorae, quando este organismo fica exposto por tempo prolongado. Embora as ostras sejam animais eurialinos e osmoconformadores, as variações bruscas de 


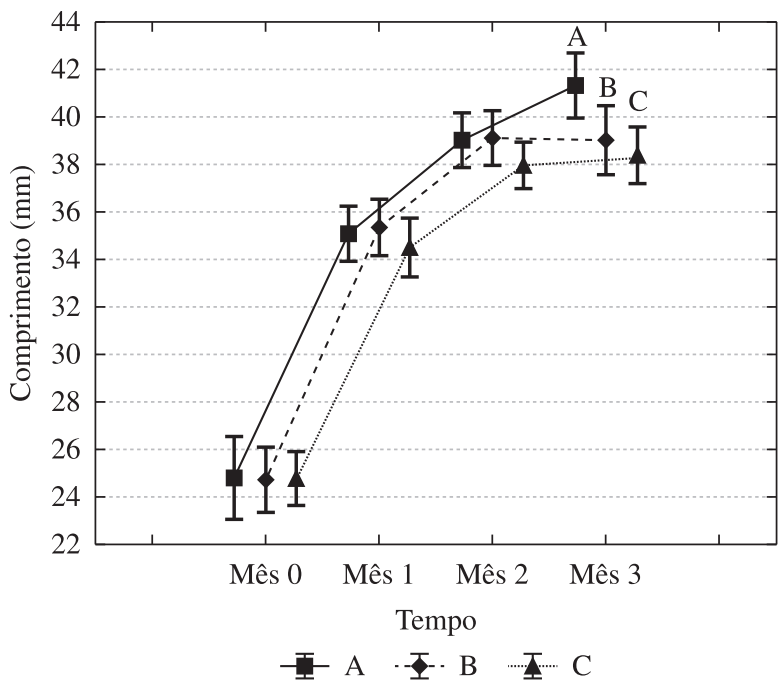

Figura 3. Comprimento ( $\mathrm{mm}$ ) médio e desvio padrão das ostras cultivadas nos tratamentos A, B e C durante os meses de cultivo.

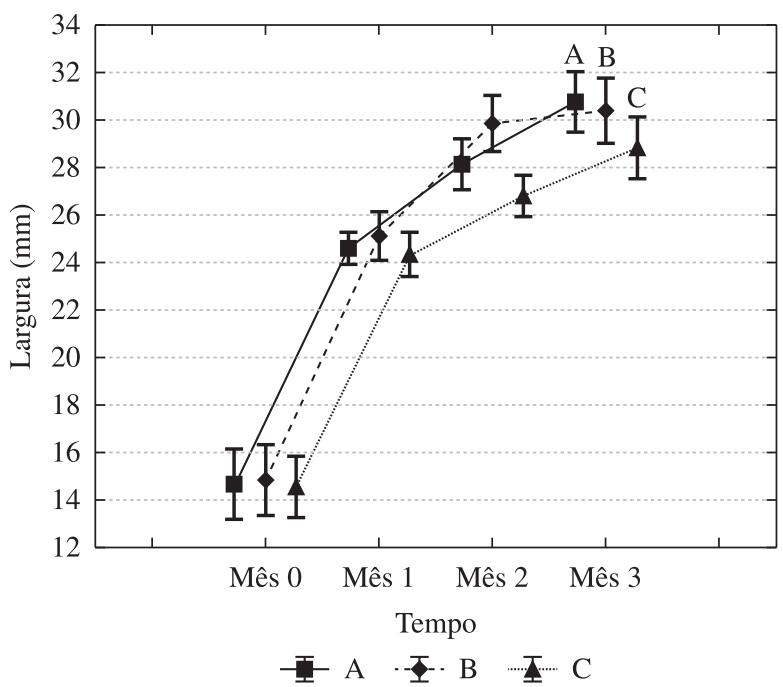

Figura 4. Largura (mm) média e desvio padrão das ostras cultivadas nos tratamentos A, B e C durante os meses de cultivo.

salinidade demandam um gasto energético que poderia ser usado para o crescimento (GUZENSKI, 1996).

Guimarães et al. (2008), estudando a influência da salinidade na sobrevivência das ostras, encontraram valores significativamente mais elevados de mortalidade em 30 e 35 de salinidade. Modesto et al. (2010) observaram mortalidades das ostras Crassostrea rhizophorae em salinidade acima de 40 e sugerem que o cultivo de C. rhizophorae deve ser realizado em áreas estuarinas com variação de salinidade entre 15 e 25.

No presente trabalho, a sobrevivência final das ostras foi de $30,4 \%$, no tratamento B $\left(800\right.$ ostras $\left./ \mathrm{m}^{2}\right)$, de $28 \%$, no tratamento A $\left(400\right.$ ostras $\left./ \mathrm{m}^{2}\right)$ e de $23,2 \%$ no tratamento C $\left(1200\right.$ ostras $\left./ \mathrm{m}^{2}\right)$. Costa (1983) considera satisfatória uma sobrevivência de apenas $7,5 \%$ a $10 \%$, para um empreendimento de cultivo de ostra, partindo de animais com tamanho médio inicial de $30 \mathrm{~mm}$, visando sua industrialização.
Pereira e Chagas Soares (1996), na região estuarina de Cananeia, cultivando $C$. brasiliana com tamanho médio inicial de 19,80 mm, em 12 meses, obtiveram um sobrevivência média final de $30,12 \%$, demonstrando viabilidade da implantação da ostreicultura na região estudada.

Moroney e Walker (1999) observaram que C. virginica, cultivada na Georgia (EUA), obtiveram uma sobrevivência máxima de 32\%, enquanto Pereira et al. (2001), na região lagunar estuarina de Cananeia, cultivando Crassostrea brasiliana, obtiveram uma sobrevivência média de $74,44 \%$. Já Modesto et al. (2010) encontraram sobrevivência final em três meses de cultivo de $44,6 \%, 27,6 \%$ e $16,6 \%$ nas densidades de 250 ostras $/ \mathrm{m}^{2}, 500$ ostras $/ \mathrm{m}^{2}$ e 750 ostras $/ \mathrm{m}^{2}$, respectivamente.

Os dados de sobrevivência obtidos no experimento estão semelhantes aos encontrados por Modesto et al. (2010) na densidade de 500 ostras $/ \mathrm{m}^{2}$ (PEREIRA; CHAGAS SOARES, 1996; MORONEY; WALKER, 1999), porém diferem dos encontrados por Pereira et al. (2001) e Costa (1983).

A densidade de cultivo é um dos principais fatores que influenciam a sobrevivência e taxa de crescimento em ostras (HONKOOP; BAYNE, 2002; BISHOP; HOOPER, 2005; MacCACCHERO; FERREIRA; GUZENSKI, 2007). No presente experimento, foram encontradas diferenças significativas para as variáveis produtivas em relação às densidades de estocagem, sendo a densidade de 400 ostras $/ \mathrm{m}^{2}$ (Tratamento A) mais recomendada.

Para melhorar as variáveis produtivas em cultivo de ostras, é necessário um menor período entre os manejos realizados, já que a limpeza das ostras é um fator de extrema importância para o sucesso do cultivo, pois o acúmulo de material em suspensão e a presença de predadores e competidores que se fixam sobre as estruturas que servem ao cultivo e nelas próprias, competem por espaço e/ou alimento.

\section{Conclusões}

Para cultivo da ostra Crassostrea rhizophorae em sistema de balsa, a densidade de 400 ostras $/ \mathrm{m}^{2}$ apresentou o melhor resultado de produção.

\section{Referências}

AKABOSHI, S.; CHAGAS SOARES, F. Cultivo experimental de Crassostrea brasiliana (Lamarck, 1819) no Canal da Bertioga, São Paulo (2354'30'S; 44¹3'42'W). Boletim do Instituo de Pesca, v. 15 , n. 1 , p. 55-65, 1988.

ALBUQUERQUE, M. C. P.; FERREIRA, J. F. Eficiência comparada do cultivo da vieira Nodipecten nodosus (Linnaeus, 1758) (Bivalvia: Pectinidae) em diferentes densidades e profundidades. Biotemas, v. 19, n. 2, p. 37-45, 2006.

BISHOP, M. J.; HOOPER, P. J. Flow, stocking density and treatment against Polydora spp.: influences on nursery growth and mortality of the oysters Crassostrea virginica and $C$. ariakensis. Aquaculture, v. 246, p. 251-261, 2005. http://dx.doi.org/10.1016/j. aquaculture.2005.01.021 
CENTRO DEPESQUISA E GESTÃODERECURSOS PESQUEIROS DO LITORAL NORDESTE - CEPENE. Boletim da estatística da pesca marítima e estuarina do Nordeste do Brasil - 2006. Tamandaré: CEPENE, 2008. 385 p.

COSTA, P. F. Manual de maricultura. Cabo Frio: Instituto de Pesquisas Marinhas 1983. 40 p.

FOOD AND AGRICULTURE ORGANIZATION THE UNITED NATIONS - FAO. The state of world fisheries and aquaculture in 2003. Rome: FAO, 2004. 153 p.

FOOD AND AGRICULTURE ORGANIZATION THE UNITED NATIONS - FAO. The state of world fisheries and aquaculture in 2008. Rome: FAO, 2009. 196 p.

FOOD AND AGRICULTURE ORGANIZATION THE UNITED NATIONS - FAO. The state of world fisheries and aquaculture 2009. Rome: FAO, 2010. 218 p.

GALTSOFF, P. S. The american oyster Crassostrea virginica (Gmelin, 1971). United Station: Fish and Wildlife Service, 1964. 64 p.

GUIMARÃES, I. M. et al. Influência da salinidade sobre a sobrevivência da ostra-do-mangue, Crassostrea rhizophorae. Arquivos de Ciências do Mar, v. 41, n. 1, p. 118-122. 2008.

GUZENSKI, J. Comparação do efeito da salinidade e concentração de substâncias húmicas no crescimento de Crassostrea rhizophorae (Guilding, 1828). 1996. 101 f. Dissertação (Mestrado em Aquicultura)-Universidade Federal de Santa Catarina, Santa Catarina, 1996.

HONKOOP, P. J. C.; BAYNE, B. L. Stocking density and growth of the pacific oyster (Crassostrea gigas) and the Sydney rock oyster (Saccostrea glomerata) in port stephens, Australia. Aquaculture, v. 213, p. 171-186, 2002. http://dx.doi.org/10.1016/S00448486(02)00030-3

IMAI, T. The Evolution of the oyster culture. In: ROTTERDAM, A. A. (Ed.). Aquaculture in shallow sea. Balkema: ASS, 1977. p. $115-262$.
JOHNSCHER-FORNASARO, G. Observações sobre populações da ostra Crassostrea rhizophorae (Guilding, 1828) em ambientes ecologicamente diferentes do litoral do estado de São Paulo. 1981. 163 f. Dissertação (Mestrado)-Universidade de São Paulo, São Paulo, 1981.

LITTLEWOOD, D. T. J. Oyster cultivation in the Caribe with emphasis on mangrove oysters in Jamaica. World Aquaculture, v. 122 , p. 70-73, 1991.

MacCACCHERO, G. B.; FERREIRA, J. F.; GUZENSKI, J. Influence of stocking density and culture management on growth and mortality of the mangrove native oyster Crassostrea sp. in southern Brazil. Biotemas, v. 20, n. 3, p. 47-53, 2007.

MEDEIROS, C. Q.; KJERFVE, B. Hydrology of a tropical estuarine system: Itamaracá, Brazil. Estuarine, Coastal and Shelf Science v. 36, p. 495-515, 1993. http://dx.doi.org/10.1006/ecss.1993.1030

MODESTO, G. A. et al. Utilização de Crassotrea rhizophorae (guilding, 1828) no tratamento dos efluentes do cultivo de Litopenaeus vannamei (Boone, 1931). Pan-American Journal of Aquatic Sciences, v. 5, p. 367-375, 2010.

MORONEY, D. A.; WALKER, R. L. The effects of the tidal and bottom placement on the growth, survival and fouling of the eastern oyster. Journal of the World Aquaculture Society, v. 30, n. 4, p. 433-442. 1999. http://dx.doi.org/10.1111/j.1749-7345.1999. tb00991.x

PEREIRA, O. M.; CHAGAS-SOARES, F. Análise da criação de ostra Crassostrea brasiliana (LAMARK, 1819), no sítio Guarapari, na região lagunar-estuarina de Cananéia - SP. Boletim do Instituto de Pesca, v. 23, p. 135-142, 1996.

PEREIRA, O. M. et al. Crescimento da ostra Crassostrea brasiliana semeada sobre tabuleiro em diferentes densidades na região estuarino-lagunar de Cananéia-SP $\left(25^{\circ} \mathrm{S}\right.$ e $\left.48^{\circ} \mathrm{W}\right)$. Boletim do Instituto de Pesca, v. 27, n. 2, p. 163-174, 2001.

SANTOS, J. J. Aspectos da ecologia e biologia da ostra Crassostrea rhizophorae (Guilding, 1828) na Bahia de Todos os Santos. 1978. 166 f. Tese (Doutorado)-Instituto de Biociências, Universidade de São Paulo, São Paulo, 1978. 